\title{
Hippocampal Function and Spatial Memory: Evidence From Functional Neuroimaging in Healthy Participants and Performance of Patients With Medial Temporal Lobe Resections
}

\author{
Véronique D. Bohbot \\ Douglas Hospital Research Centre and McGill University
}

\author{
Giuseppe Iaria \\ Douglas Hospital Research Centre, McGill University, \\ Università di Roma "La Sapienza," and Instituto di Ricovero e \\ Cura a Carattere Scientifico (IRCCS) Fondazione Santa Lucia
}

\author{
Michael Petrides \\ McGill University
}

\begin{abstract}
Several strategies can be used to find a destination in the environment. Using a virtual environment, the authors identified 2 strategies dependent on 2 different memory systems. A spatial strategy involved the use of multiple landmarks available in the environment, and a response strategy involved right and left turns from a given start position. Although a probe trial provided an objective measure of the strategy used, classification that was based on verbal reports was used in small groups to avoid risks of misclassification. The authors first demonstrated that the spatial strategy led to a significant activity of the hippocampus, whereas the response strategy led to a sustained activity in the caudate nucleus. Then, the authors administered the task to 15 patients with lesions to the medial temporal lobe, showing an impaired ability using the spatial strategy. Imaging and neuropsychological results are discussed to shed light on the human navigation system.
\end{abstract}

Navigation in the environment and topographical learning have been studied in human participants for over 100 years (Badal, 1888). Topographical learning has been defined as learning the way from one place to another (Hecaen, Tzortzis, \& Rondot, 1980). Its biological relevance becomes evident when one encounters patients with brain damage, for whom leaving the house is a major challenge because they fear getting lost.

One way to find a destination in the environment is to establish in memory a cognitive map of the environment. Such an allocentric spatial strategy relies on building knowledge of the relationship among multiple environmental landmarks, irrespective of the position of the observer (O'Keefe \& Nadel, 1978). This strategy can be distinguished from route learning, which can also be used to find destinations in the environment and involves a "stimulus-

Véronique D. Bohbot, Douglas Hospital Research Centre, Verdun, Quebec, Canada; and Department of Psychiatry, McGill University, Verdun. Giuseppe Iaria, Douglas Hospital Research Centre; Department of Psychiatry, McGill University, Verdun; Montreal Neurological Institute, McGill University, Montreal, Quebec, Canada; Dipartimento di Psicologia, Università di Roma "La Sapienza," Rome, Italy; and IRCCS Fondazione Santa Lucia, Rome. Michael Petrides, Montreal Neurological Institute, McGill University, Montreal.

We thank Joelle Crane and Brook Thorndycraft for their help in recruiting participants and collecting data. This study was supported by funds awarded to Véronique D. Bohbot, including Natural Sciences and Engineering Research Council of Canada Grant 239920, Canadian Institutes of Health Research Grant 64381, Fond de la Recherche en Santé du Québec Grant 3234, and start-up funds from the Douglas Hospital Research Centre.

Correspondence concerning this article should be addressed to Véronique D. Bohbot, Department of Psychiatry, McGill University, Frank B. Common Building, 6875 Boulevard LaSalle, Verdun, Quebec H4H 1R3, Canada. E-mail: veronique.bohbot@mcgill.ca response-stimulus" chain leading to a goal (p. 81). These distinctions are based on several decades of research dating back to the 1930s, influenced by the works of Hull, Watson, Lashley, Tolman, and Hebb (O'Keefe \& Nadel, 1978). The work by Milner and her colleagues suggested the existence of multiple memory systems in the mammalian brain (B. Milner, 1972). By 1978, O'Keefe and Nadel had published a theory of hippocampal function proposing that learning to navigate to a place in the environment on the basis of these two strategies depends on different neural structures and that the hippocampal system underlies the formation of a cognitive map (O'Keefe \& Nadel, 1978).

The theory of O'Keefe and Nadel (1978) that the hippocampal system is critical for the establishment of a cognitive map of the environment was influenced by the discovery that there are place cells in the hippocampus of rats, recorded as the rats were navigating in the environment (O'Keefe \& Dostrovsky, 1971). Place cells discharged when the rat was in a specific location. In addition, lesions to the hippocampus in rats were shown to lead to severe spatial learning and memory deficits (Jarrard, 1993; Morris, Garrud, Rawlins, \& O'Keefe, 1982; Olton \& Papas, 1979). The hippocampus in monkeys has also been shown to have locationspecific neurons that fire when the monkey moves to a certain location in the environment or looks at a specific part of it (Matsumura et al., 1999; Rolls, Robertson, \& Georges-Francois, 1997). Furthermore, there is evidence from lesion studies with monkeys that the hippocampus is involved in memory for spatial information, such as complex naturalistic scenes (Gaffan, 1992).

The first clear reports implicating the hippocampus in learning and memory in humans came from studies of patients with bilateral medial temporal lobe damage (Scoville \& Milner, 1957). These patients experienced a profound learning deficit for facts and events, whereas other forms of learning, such as skills, habits, conditioning, and priming, were preserved (B. Milner, 1972; Sco- 
ville \& Milner, 1957; Vargha-Khadem et al., 1997; Zola-Morgan, Squire, \& Amaral, 1986). The deficit resulting from damage to the hippocampus includes spatial learning and memory (Abrahams et al., 1999; Bohbot et al., 1998; Maguire, Burke, Phillips, \& Staunton, 1996; Nunn, Graydon, Polkey, \& Morris, 1999; Pigott \& Milner, 1993; Smith \& Milner, 1989). More important, place fields have now been recorded in the human hippocampus, which provides important support to the cognitive map theory (Ekstrom et al., 2003).

The term place learning is used to refer to learning in tasks in which locations in the environment can be reached with the use of various cognitive strategies (e.g., spatial learning, response learning, or both) that can be dependent on different memory systems (Eichenbaum, Stewart, \& Morris, 1990; McDonald \& White, 1995). In this article, we use the term spatial learning to refer to learning that requires knowledge of the relationship between multiple environmental landmarks as defined by O'Keefe and Nadel (1978), in other words, relational learning (Eichenbaum, Schoenbaum, Young, \& Bunsey, 1996; McDonald \& White, 1995) applied to the spatial domain. We use the term response learning to refer to learning that is acquired through the repetition of rewarded responses to stimuli (Packard \& Knowlton, 2002; White \& McDonald, 2002). Knowledge about the relationship among different items in the environment leads to a more flexible approach to finding one's way in the environment compared with learning a sequence of specific behavioral responses and allows individuals to derive novel trajectories between various locations in a direct path.

In a study from our laboratory, we tested participants on a place-learning task, the four on eight virtual maze that allows for both spatial and nonspatial response strategies (Iaria, Petrides, Dagher, Pike, \& Bohbot, 2003). Feedback from the participants and a probe test were used to dissociate the two strategies. Yet all participants were tested in the same virtual environment and given the same instructions. We found that $50 \%$ of the participants spontaneously used a spatial strategy at the beginning of the experiment, and the other $50 \%$ used a nonspatial strategy (Iaria et al., 2003). We further found that $40 \%$ of spatial learners switched to a nonspatial strategy. In the present study, we first tested 15 patients with unilateral excisions from the medial temporal lobes to investigate further the involvement of the hippocampus in spatial memory. Then, we presented functional MRI (fMRI) data, confirming the involvement of the hippocampus when healthy human participants are engaged in a spatial strategy while navigating in the environment. Neuropsychological and imaging data are discussed to shed light on the role of the hippocampus in place learning and spatial memory.

\section{Method}

\section{Experiment 1}

Participants. Fifteen patients who had undergone a unilateral excision from the medial temporal lobe and 10 healthy control participants took part in this study. Informed consent was obtained in a manner approved by the local ethics committee.

Control group. Ten control participants who were free from any neurological conditions were selected to participate in this study. They were chosen to be as similar as possible to the patients with medial temporal lobe lesions in education, age, and gender. The group included 4 men and 6 women, ranging from 36 to 53 years of age $(M$ age $=43.0$ years, $S D=5.9$ years). Participants had finished from 13 to 18 years of education $(M$ $=15.3$ years, $S D=1.4$ years), and all but 1 participant were right handed.

Medial temporal lobe group. Fifteen patients who had previously undergone brain surgery at the Montreal Neurological Hospital (Montreal, Quebec, Canada) to alleviate pharmacologically intractable epilepsy or to remove a congenital vascular malformation participated in this study. All but 3 of these patients were right handed. There were 6 men and 9 women, ranging from 24 to 57 years of age ( $M$ age $=42.5$ years, $S D=8.7$ years). The patients had completed from 11 to 18 years of education $(M=14.7$ years, $S D=2.5$ years) and had Wechsler (Wechsler, 1955) full scale IQ scores ranging from 81 to $122(M$ score $=97.9, S D=12.8)$. They were tested within 1 to 10 years of their last surgery $(M=3.70, S D=2.89)$.

Resections. All patients received one of the following types of surgery: anterior temporal resection ( $n=6$ on the right side and $n=3$ on the left side), selective amygdalo-hippocampectomy ( $n=5$ on the right side), or a small lesionectomy ( $n=1$ on the right side). The anterior temporal resection typically involved damage to the hippocampus, amygdala, and entorhinal and perirhinal cortical regions, as well as damage to the temporal pole and to the superior, middle, and inferior temporal cortices. The selective amygdalo-hippocampectomy involved damage to the hippocampus and amygdala with partial damage to other structures, such as the middle temporal gyrus, entorhinal cortex, and perirhinal cortex. The 1 patient with a lesionectomy obtained a resection of the posterior part of the temporal lobe, lateral to the hippocampus and parahippocampal cortex.

Task. A computer game (Unreal Tournament, Epic Games Inc., Raleigh, NC) was used to create a navigation task that took place in a virtual environment. Participants performed the task using a personal computer. The virtual environment consisted of a landscape (mountains and sunset), two trees, and a short wall located between the landscape and the trees. At the center of the environment, there was an eight-arm radial maze with a central starting location. At the end of each arm, a staircase led down to a chamber in which an object could be retrieved. The participants started each trial on the central platform and could see the location of the objects only by going down the arms.

To move within the environment, the participants used a keypad with forward, backward, left turn, and right turn buttons. Before testing, the participants were asked to spend a few minutes moving around a virtual room to practice the motor aspects of the task. The experiment started when the participants felt comfortable using the keypad.

The task was composed of two parts. In Part 1, four of the eight arms were accessible with objects at the end of each arm; in Part 2, all arms were accessible and objects were present at the end of the four arms that had been blocked in Part 1. Participants were required to retrieve all four objects from the accessible arms in Part 1. They were then asked to remember which arms they had visited in order to avoid them and retrieve the other four objects in Part 2. An error consisted of an entry into an arm that did not contain an object.

Three types of trials were administered. In Part 1 of Trial Type A (Sequence A), Arms 1, 3, 4, and 6 were accessible and contained an object; in Part 2, the four identical objects were located at the end of the four arms that had previously been blocked (i.e., Arms 2, 5, 7, and 8). The sequence of accessible arms was different in Trial Type B (Sequence B): In Part 1, Arms 2, 3, 7, and 8 were open, and in Part 2, the objects were located at the end of Arms 1, 4, 5, and 6. Trial Type C (Sequence C) was a probe trial. In Part 1, this trial was identical to the Trial Type A (Sequence A); however, in Part 2 the walls around the radial maze were raised to conceal the landscape, and the trees were removed so that no landmarks were available. Moreover, eight objects were present (one at the end of each arm). For this and every trial, participants finished the trial after retrieving four objects. The rationale of the probe trial was the following: If participants used a strategy that was based on memory for landmarks, this change 
in the environment should result in an increase in errors. On the other hand, if participants did not rely on the environmental landmarks, no increase in errors should occur.

Testing was divided into three consecutive sections. In Section 1 (four trials), the sequence of trials was A, B, A, C. In Section 2, the participants repeated Trial Type A five times, which served as a training phase. Finally, Section 3 was identical to Section 1 (i.e., Trial Types A, B, A, C). We recorded all the errors as well as the time the participants spent performing the trials.

At the end of the experiment, the participants were asked to report how they had solved the task from the beginning to the end of the experiment. Moreover, participants were asked if they remembered whether the starting position was the same or different at every trial, if they did not mention it spontaneously. Participants were categorized as having used a nonspatial strategy if they associated the arms with numbers or letters or if they counted the arms (clockwise or counterclockwise) from a single starting point. If participants had solved the task by using at least two landmarks and had not mentioned a nonspatial strategy, they were categorized as using spatial memory. Participants who mentioned using spatial memory at the beginning and later shifted to the nonspatial strategy were placed into the "shift" group. The verbal reports were preferred over the probe trial to classify participants according to their strategy for the following reason. Occasionally, participants using the nonspatial strategy counted from a single landmark. Because all landmarks were removed during the probe trial, these participants would likely make errors, behaving as if they used a spatial strategy. Therefore, there is a risk for misclassification in participants who showed errors on the probe trial but who claimed to have counted from a single landmark if the probe errors alone are used for grouping participants. Possible misclassifications would be particularly detrimental in studies with small groups of participants; therefore, the verbal classification method was favored in this case.

\section{Experiment 2}

Participants. We tested 14 young right-handed participants who had no history of neurological disorders ( 7 men and 7 women matched for age and education; $M$ age $=25.3$ years, $S D=2.8$ years). Informed consent was obtained in a manner approved by the local ethics committee.

Task. The task and the virtual environment were identical to those used in Experiment 1. In Part 1, four of the eight arms were accessible with objects at the end of each arm; in Part 2, all arms were accessible and four objects were present in the four arms that had been blocked in Part 1 . The participants were asked to retrieve all four objects from the accessible arms in Part 1 and to remember which arms were visited to avoid these and find the four objects in Part 2. As in Experiment 1, there were three trial types (A, B, and C). In this fMRI study, however, there was an additional visuomotor control task during which the participants were asked to pick up the same objects randomly placed at the end of four arms. This time, the objects were visible from the center of the maze. Because of time constraints, fewer trials were administered in this fMRI experiment compared with Experiment 1. We administered eight scans (otherwise called runs) of $7 \mathrm{~min}$ each. In each scan, the participants performed one experimental trial (A, B, or C) and several visuomotor control trials, linked to one another until the end of the 7-min scan. The following order of trials was performed by the participants: A, B, C, A, A, A, B, and C. Before scanning, as in Experiment 1, the participants spent a few minutes moving in a virtual room that was different from the experimental environment to practice the motor aspects of the task. At the end of the experiment, the participants were debriefed using the same procedure adopted in Experiment 1 . The verbal report was used for the classification of participants for the same reasons as those outlined in Experiment 1. We recorded all the errors as well as the time the participants spent performing the trials.

MRI acquisition data. The experiment consisted of eight scans (7 min each). In each scan, before the experimental and visuomotor control conditions, the participants performed a task identical to the visuomotor control with the exception that there was one visible object instead of four. This allowed us to control for equilibration effects by excluding the first few frames of each scan from the analysis. Moreover, because of the variability among participants in the time taken to perform the tasks, we used homemade software to record frame times, every keystroke made by the participants, as well as the keystrokes by the experimenter indicating transition from one task to another. This allowed us to exclude from the analysis the frames acquired during the translations between the tasks. The fMRI scans were obtained with a Siemens Vision 1.5T system (Siemens AG, Erlangen, Germany). For the anatomical images, a three-dimensional gradient echo acquisition was used to collect 80 contiguous, 2-mm T1weighted images in the sagittal plane. The functional scanning session began with a sagittal localizer followed by a series of blood oxygen level-dependent (BOLD) scans. Each functional scan was acquired using 26 contiguous, 5-mm axial slices, positioned parallel to the hippocampus and covering the entire brain $(64 \times 64$ matrix; echo time $[\mathrm{TE}]=50$ $\mathrm{ms}$; number of frames $=105$; time between measurements $=4 \mathrm{~s}$; field of view $[\mathrm{FoV}]=320 \mathrm{~mm})$. BOLD signal images were spatially smoothed (6-mm Gaussian kernel), corrected for motion, and linearly transformed into standard stereotaxic space (Talairach \& Tournoux, 1988) using inhouse software (Collins, Neelin, Peters, \& Evans, 1994). Individual $t$ statistic maps of the comparisons between experimental and control tasks in each scan, as well as group-averaged statistical images and correlation maps, were obtained using the FMRISTAT software package (Worsley et al., 2002). The $t$-statistic thresholds corrected for multiple comparisons for the whole brain volume were $t=4.43, p<.05$, and $t=5.25, p<.001$. For the predicted searches, the corrected thresholds were determined to be $t=3.25, p<.05$, and $t=4.30, p<.001$, on the basis of the sum of the volumes of the right hippocampus and the right caudate nucleus $\left(3,500+5,500 \mathrm{~mm}^{3}\right.$, respectively). For the correlation analyses, the uncorrected threshold for the predicted searches (in hippocampus and in the caudate nucleus) was $t=1.96, p<.05$. The threshold corrected for multiple comparisons for the whole-brain volume, $t=4.43, p<.05$, was used for other brain areas for the correlation analyses.

\section{Results}

\section{Experiment 1}

The proportion of patients who spontaneously used one strategy over the other is comparable with that of young healthy participants (Iaria et al., 2003). The debriefing reports indicated that, at the beginning of the experiment (Section 1), 9 patients with damage to the medial temporal lobe $(60 \%)$ performed the task using the relationships between landmarks available in the environment (spatial memory), and 6 patients performed it by counting the arms clockwise or counterclockwise from a single point (nonspatial strategy). All 6 patients who initially used the nonspatial strategy had right-sided excisions (1 patient had an anterior temporal resection, 1 patient had a lesionectomy, and 4 patients had a selective amygdalo-hippocampectomy). Three of the patients who used spatial memory spent three times longer than all other patients in terms of the time it took to complete the first section. These patients had undergone an anterior temporal resection on the right side and a left and a right amygdalo-hippocampectomy. They took longer to perform the task because they made many more errors than other patients, with an average of 14.9 errors per trial in the four trials of Section 1, relative to the 8.1 errors for the nonspatial patients. Consequently, testing was stopped after the completion of Section 1 for these 3 patients, and they were removed from subsequent analysis. The other 12 patients continued until the end 
of the experiment. By the end of the test, 10 patients were using the nonspatial strategy and only 2 of the patients were using spatial memory. Thus, with practice 4 of the 9 patients (45\%) who were initially using spatial memory shifted to using a nonspatial strategy; in other words, they first used environmental landmarks to orient themselves and later relied on counting the arms from a single starting point. Two of the patients in the shift group had a left anterior temporal resection, 1 patient had an anterior temporal resection on the right, and the other patient had a right-sided amygdalo-hippocampectomy. The 2 patients who used spatial memory from the start to the end of the experiment had right-sided resections: a selective amygdalo-hippocampectomy and an anterior temporal lobe resection. All patients made at least 1 error in the probe trial of Sections 1 and 3. Therefore, the probe trial could not be used to discriminate between the two strategies. However, this could indicate that patients using nonspatial strategies may have counted arms from a single landmark in the environment instead of using the starting position.

Because of the low number of participants and the possible violation of normal distribution assumption, the results were analysed first with nonparametric statistics. If the normality test and the equal variance test were passed, further testing to evaluate differences between the groups was done with Tukey's honestly significant difference (HSD) test. The comparison of interest in this experiment involved the spatial memory and nonspatial strategy medial temporal lobe groups. We expected that patients with medial temporal lobe damage who used the nonspatial strategy would perform better than patients with medial temporal damage using spatial memory. Therefore, the medial temporal lobe nonspatial strategy group served as the ideal comparison group because it was similar to the medial temporal lobe spatial memory group in type of disorder, brain damage, quality of life, sex, age, and education. We therefore chose to limit statistical comparisons to the spatial memory versus nonspatial strategy medial temporal lobe groups. Along these lines, only the nonspatial strategy healthy control group $(n=7)$ was used for comparisons with the nonspatial strategy, medial temporal lobe-damaged group. The single control participant who used a spatial strategy and the 2 control participants who shifted from spatial memory to a nonspatial strategy performed similarly to the nonspatial strategy healthy control groups, with an average of 0.40 and 0.30 errors, respectively, in Section 2; these participants made an average of 0.10 and 1.25 errors, respectively, in Section 3 .

There was a significant difference across all groups in the number of errors made while performing the A type trials in the four on eight virtual maze (Kruskal-Wallis $H=33.43, d f=11$, $p<.001)$. Further pairwise comparisons were carried out with Tukey's HSD test. The medial temporal lobe spatial memory group was significantly impaired relative to the medial temporal lobe nonspatial strategy group on errors made in Sections 2 and 3 (Section 2: $q=5.296, p<.05$; Section 3: $q=5.522, p<.05$ ), but the groups were not significantly different in Section $1(q=0.399$, $p>.05, n s)$. The medial temporal lobe nonspatial strategy group performed similarly to the nonspatial strategy healthy control group on all sections (Section 1: $q=1.841, p>0.05$, $n s$; Section 2: $q=0.006, p>.05, n s$; Section 3: $q=0.516, p>.05$; see Figure 1).

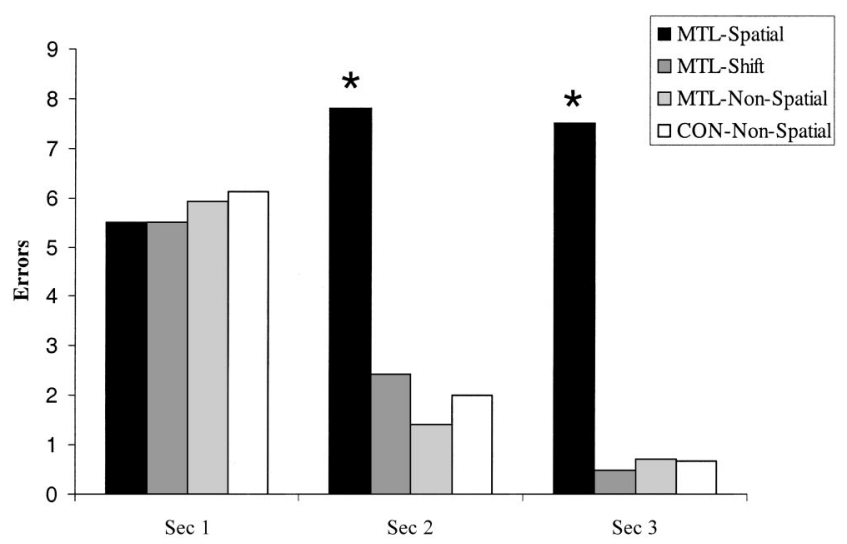

Figure 1. Average errors made by participants with medial temporal lobe (MTL) excisions and control (CON) participants on the standard Trial Type A of the four on eight virtual task. Participants were grouped in terms of the strategy used during performance of the four on eight virtual task (spatial, shift, and nonspatial). Errors are shown for Sections (Sec) 1 through 3 . The 1 control participant who used a spatial strategy and the 1 control participant who shifted from spatial memory to a nonspatial strategy performed similarly to the nonspatial strategy healthy control groups with, on average, 0.40 and 0.30 errors, respectively, in Section 2. These participants made on average 0.10 and 1.25 errors, respectively, in Section 3. *Significant difference from the nonspatial MTL group $(p<.05)$.

\section{Experiment 2}

fMRI data. Brain regions involved in the performance of the task for the entire subject pool $(N=14)$, independent of the different strategies spontaneously adopted to solve the task, have been described elsewhere (Iaria et al., 2003). In brief, compared with the visuomotor control task, we found a statistically increased BOLD signal during the performance of the experimental task bilaterally in the posterior parietal cortex (Area 7), the putamen, the right caudate nucleus, left middle occipital gyrus, and the right cerebellum. In addition, we found bilateral activity of the middorsolateral prefrontal cortex (Area 9/46), primary motor cortex (Area 4), and the supplementary motor cortex (Area 6), extending into the adjacent right cingulate motor region.

Then we analyzed the fMRI data of the spatial memory group $(n=7)$ separately from that of the nonspatial strategy group $(n=$ 7) to investigate our hypothesis that the hippocampal and striatal systems would be differentially involved depending on the navigational method adopted by the participant and the practice in performing the task. The experimental and probe conditions were contrasted with the control condition that was performed in every scan. In the spatial memory group, there was a significantly greater BOLD signal in the experimental compared with the control condition in the right hippocampus in the first (see Figure 2; $[x=32$, $y=-14, z=-20], t=4.41)$ and second scans $(x=22, y=-16$, $z=-14), t=3.49$. In contrast, the nonspatial strategy group showed no activity increase in the hippocampus in any of the scans but demonstrated significant activity in the caudate nucleus in Scans $4(x=20, y=8, z=18), t=3.81 ; 5(x=14, y=-8, z=$ $22), t=4.04 ; 6(x=20, y=-24, z=22), t=4.41 ;(x=-12$, $y=-10, z=18), t=4.30 ; 7(x=10, y=-4, z=20), t=2.94$, which approached statistical significance; and $8(x=8, y=-4$, 


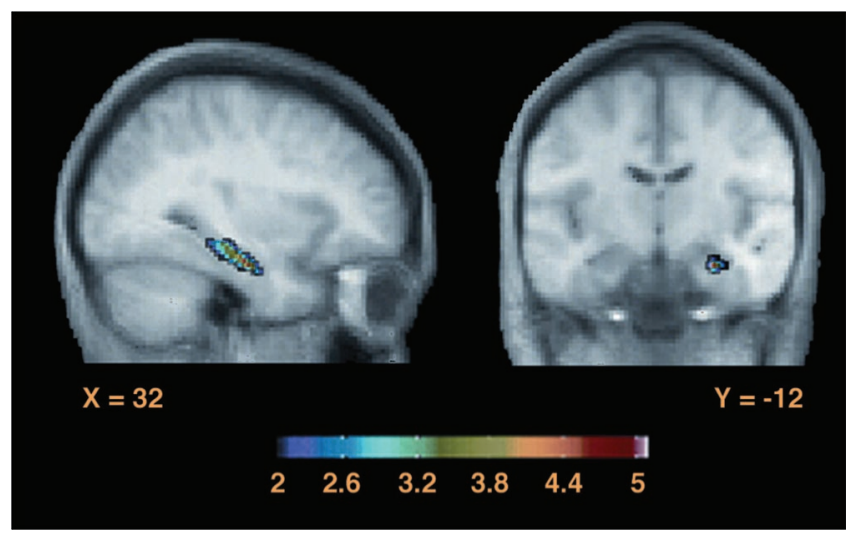

Figure 2. Activity in the hippocampus found in the spatial memory group relative to the nonspatial strategy group in healthy participants. The $t$ statistic maps are superimposed onto the anatomical average of all participants and displayed in the sagittal and coronal planes. The comparison of the experimental minus the control conditions yielded greater activity in the right hippocampus of the spatial memory group than in the nonspatial strategy group in the first $\operatorname{scan}(x=32, y=-14, z=-20), t=4.41$.

$z=20), t=5.36$. Thus, with practice, activity in the caudate nucleus emerged in this group and was sustained until the end of the experiment (i.e., Scans 4, 5, 6, 7, and 8). In the spatial memory group, activity in the caudate nucleus was inconsistent, appearing only in Scans $2(x=14, y=-6, z=20), t=4.82$, and $8(x=24$, $y=-22, z=24), t=4.65$; $(x=-20, y=-2, z=24), t=3.58$. Thus, the hippocampus was active only in the spatial memory group, and the nonspatial strategy group showed a more sustained pattern of activation in the caudate nucleus.

\section{Discussion}

In the fMRI experiment, healthy participants were tested on a place-learning task that allowed for two dissociable performance strategies. Only performance of the task by a spatial strategy, which involved learning the relationships between multiple environmental landmarks, led to a significant activation of the hippocampus. Adoption of the nonspatial strategy in performing the task did not lead to increased activity in the hippocampus. Subtraction of the fMRI signal of the nonspatial learners from that of the spatial learners on Trial 1 did not change the highly significant activation in the hippocampus. This result suggests that the hippocampus of nonspatial learners was minimally, if at all, engaged in this task. In fact, the brain activity common to all participants (spatial and nonspatial learner) did not include points of significance at the level of the hippocampus (Iaria et al., 2003). The variability in strategies used by participants could provide a potential explanation for the fact that numerous earlier functional neuroimaging studies of spatial learning have failed to show hippocampal involvement. Furthermore, in participants who initially adopted a spatial strategy and then shifted to a nonspatial strategy, the activation in the hippocampus disappeared. Thus, if participants' strategies vary across the scanning session, there will be additional variability that would wash out measures of hippocampal contribution to a task. It should be remembered that, in this experiment, the nonspatial learners were tested on the same nav- igation task and were given the same instructions. Therefore, differences in hippocampal activation must have been related to the cognitive strategy used when performing the task.

The parahippocampal cortex has been implicated in navigation in a virtual maze (Aguirre, Detre, Alsop, \& D'Esposito, 1996; Maguire, Frith, Burgess, Donnett, \& O'Keefe, 1998) and in processing and remembering scene information (Brewer, Zhao, Desmond, Glover, \& Gabrieli, 1998; Epstein \& Kanwisher, 1998; Kohler, Crane, \& Milner, 2002). It is interesting that memory disorders for wayfinding or topographical amnesia have been correlated with damage to the region of the right medial occipitotemporo-parietal cortex, especially the parahippocampal cortex (Barrash, 1998; Habib \& Sirigu, 1987; Hublet \& Demeurisse, 1992). In a navigation task that involved memory for only one location in one room, Bohbot and coworkers (1998) found that the right parahippocampal cortex was involved in allocentric spatial memory without the necessary involvement of the hippocampus. Patients with thermocoagulation lesions of the right hippocampus sparing the parahippocampal cortex in Bohbot et al. (1998) could have identified the target location in relation to the view of a single scene. Although the parahippocampal cortex is believed to be critical in the current study, the present results did not show parahippocampal activation because it would have been subtracted out by the control task that also involved navigation in the same environment, although without an intended mnemonic component.

It is likely that the parahippocampal cortex contributes to the establishment of a cognitive map of the environment by providing spatial scene information to the hippocampus. The parahippocampal cortex itself receives visuospatial information from the parietal cortex (A. D. Milner \& Goodale, 1995; Ungerleider \& Haxby, 1994; Van Hoesen, 1982). It also receives information about specific objects in the environment from the ventral occipitotemporal cortex Areas V4, TE, and TEO (Aguirre, Zarahn, \& D'Esposito, 1998; Suzuki, 1996). When memory for the locations of a larger set of objects in a three-dimensional space was required (i.e., when all the information could not be contained in a single scene), Bohbot et al. (1998) found that the right hippocampus then became critical in a study of patients with selective thermocoagulation lesions of the hippocampus, even when the parahippocampal cortex was intact. Along similar lines, King, Burgess, Hartley, Vargha-Kadhem, and O'Keefe (2002) proposed that the hippocampus is critical when viewpoint independence is necessary (King et al., 2002). These and other possible differences between the role of the parahippocampal cortex and hippocampus were suggested in a previous article (Bohbot, Allen, \& Nadel, 2000). A study supporting this point of view was published by Ekstrom et al. (2003). In this study, place-sensitive neurons were recorded from the human hippocampus and parahippocampal cortex in vivo while participants navigated in a virtual environment. It is interesting that the place fields were found in the hippocampus more than in other regions of the brain, such as the frontal cortex, amygdala, and parahippocampal cortex; whereas cells in the parahippocampal cortex responded more to views of target landmarks (as opposed to views of people or background). In summary, when memory for spatial relationships is used to build a cognitive map of the environment (O'Keefe \& Nadel, 1978), we found that the hippocampal contribution was necessary.

The fMRI study showed that adoption of the nonspatial strategy involved sustained activation of the caudate nucleus (on Trials 4, 
5, 6, and 8). As argued in Iaria et al. (2003), the repetition of this strategy is thought to lead to response learning in humans, as in rodents. Evidence supporting this point comes from the fact that the activation of the caudate nucleus on Trial 7 was below the significance threshold; whereas Trials 4, 5, 6, and 8 involved the common pattern of rewarded arms (Trial Type A, with rewards in Arms 2, 5, 7, and 8), Trial 7 involved a different pattern of rewarded arms (Trial Type B, with rewards in Arms 1, 4, 5, and 6). Therefore, the activity of the caudate nucleus in the nonspatial group is present for the repeated pattern (Trial Type A) but not when the pattern changes (Trial Type B). The use of spatial memory also involved the caudate nucleus, but it was inconsistent, appearing on Trials 2 and 8 . Trial 2 was the only other Trial Type $\mathrm{B}$, so the caudate activity was unrelated to the pattern repetition here. Trial 8 was the last trial. Because all the spatial learners shifted strategies, it is possible that the activity in caudate measured on Trial 8 is related to the use of nonspatial strategy in spatial learners.

A response in navigation involves making an approach or avoidance behavior to a single stimulus (S-R) or to a configuration of stimuli (intersection). For example, a rat can learn that it has to turn right at the intersection of a plus maze (four-arm maze) to find a reward. A route traveled by a participant to reach a distal location can be based on a series of responses by going from one landmark to another, say from Landmarks $\mathrm{A}$ to $\mathrm{B}$ and $\mathrm{B}$ to $\mathrm{C}$, without knowing the relationship between all landmarks (e.g., the relationship between $\mathrm{A}$ and $\mathrm{C}$ is unknown). In this type of response learning, responses are learned gradually; they are part of the habit system (Mishkin \& Petri, 1984; Packard \& McGaugh, 1996). In rats, there is strong evidence that the striatum or caudoputamen matrix supports response learning (White \& McDonald, 2002). In the primate brain, the caudoputamen matrix differentiates more clearly into the caudate nucleus and the putamen. It is important that the striatum is involved not only in the development of motor habits but also in cognitive S-R habit formation (Packard \& Knowlton, 2002; White \& McDonald, 2002). Rats with lesions to the fornix, which effectively disables the hippocampus, can learn the discrimination between two places in a radial maze if the views are very different (i.e., the choices are spatially separated by an angular distance of $135^{\circ}$ or $180^{\circ}$; McDonald \& White, 1995). A combined lesion to the hippocampus and striatum led to learning impairments (McDonald \& White, 1995), suggesting that the striatum is critical for learning the place response. When the two choices were at an angular distance of $45^{\circ}$, rats with fornix lesions were not able to discriminate between these two choices to obtain the reward (McDonald \& White, 1995). A rat cannot learn to discriminate between two adjacent arms without the hippocampus because there is substantial overlap between the cues with which a response would be associated (White \& McDonald, 2002). In a radial maze, when rats enter adjacent arms that are not rewarded, they can see the same cue (S), and the lack of reward will weaken the $\mathrm{S}-\mathrm{R}$ association. This is consistent with a model of $\mathrm{S}-\mathrm{R}$, in which an approach response $(\mathrm{R})$ toward a stimulus $(\mathrm{S})$ will be strengthened if it is followed by a reinforcement (White \& McDonald, 2002). The limitation of the response system appears when the relationship among several cues is required, a process highly dependent on the hippocampus.

Functional brain-imaging studies in humans often show regions of activity in the caudate nucleus, but the role of the caudate nucleus in navigation in humans has been poorly investigated. There are several findings worth noting. In the navigation study on the involvement of the hippocampus in the accuracy of new routes, a correlation of speed of decision making with fMRI activity revealed an activation of the caudate nucleus (Maguire, Burgess, et al., 1998). Although the authors did not speculate about the role of the caudate in navigation, this finding is consistent with expectations from studies with rats. Two recent studies specifically outlined the role of the caudate nucleus in navigation (Hartley, Maguire, Spiers, \& Burgess, 2003; Iaria et al., 2003). The caudate nucleus was found to be active in an fMRI paradigm when subjects were asked to follow a specified route. There was a positive correlation between speed in following a route and fMRI activation of the caudate nucleus. Also, subjects who activated the caudate nucleus during wayfinding more than during route following navigated less well in the wayfinding condition than subjects who showed the reverse pattern (Hartley et al., 2003). A key finding, in agreement with the data presented here, was that hippocampal activation positively correlated with wayfinding accuracy.

Results from our laboratory showed sustained activation of the caudate nucleus in participants using a nonspatial strategy as opposed to the sporadic activity noted in spatial learners. Furthermore, there was a significant negative correlation between fMRI activity of the caudate nucleus and performance measures such as latency and errors (i.e., the activity of the caudate nucleus increased as participants took less time and made fewer errors; Iaria et al., 2003).

Massive projections from the parahippocampal cortex to the caudate nucleus have been demonstrated in monkeys (Suzuki, 1996). If these findings can be extended to humans, it would mean that the human caudate nucleus receives scene information. This would support the role of the caudate nucleus in navigation. The scene would serve as a stimulus that would get participants engaged in a response. A lesion to the parahippocampal cortex would therefore impair recognition of scenes, but more important, this lesion would deprive both the hippocampal and caudate nucleus memory systems from scene information. This could be a reason why the most profound cases of environmental or topographical amnesia involve a lesion to the parahippocampal cortex in humans. Therefore, the parahippocampal cortex plays a pivotal role in navigation by providing information about scenes to both the hippocampal and caudate nucleus memory systems.

The critical contribution of the hippocampus in spatial memory was further confirmed in the study involving patients with damage to the medial temporal lobe (Experiment 1). Like healthy participants (Iaria et al., 2003), the patients with lesions to the medial temporal lobes used both spatial and nonspatial strategies. The patients using spatial strategies made significantly more errors than those who used the nonspatial strategy. Furthermore, patients with medial temporal lobe lesions using nonspatial strategies were no different from age- and education-matched control participants who also used nonspatial strategies. The finding that patients who have a compromised hippocampus would be impaired at a spatial task is not surprising. However, the fact that over half of the patients with damage to the medial temporal lobes would spontaneously use the spatial strategy was unforeseen. Although these people may have the potential to compensate for their spatial 
deficit by using the nonspatial strategy that is dependent on other parts of their brain, they do not necessarily do so.

Careful analysis of the participants' reports indicated that some of the patients confused several cues. Another patient who used the nonspatial strategy did not think of counting the arms from a given start position. Instead, this patient entered one arm after the other in a clockwise fashion, including the arms that did not contain objects, therefore leading to errors. If the hippocampus is not involved in the nonspatial strategy, as indicated by the fMRI study, there may be significant potential for wayfinding therapy in the participants whose response-learning pathway is intact.

The fact that half of the patients with a compromised hippocampus spontaneously chose to use a spatial strategy and were impaired at doing so deserves some attention. About half of the participants using spatial memory shifted to the nonspatial strategy. The fact that the other half continued using the inefficient spatial memory strategy is intriguing. There are several possibilities to explore: (a) The spontaneous use of one strategy is a random process, (b) there may be genetic predispositions for using a given strategy, or (c) there could be an experience-dependent bias to use a given strategy. It should be acknowledged that the choice to use either spatial or nonspatial strategies could have been random. The use of spatial memory would still lead to errors in patients with lesions to the hippocampus and to a significant peak in the control participants during fMRI. Although people who switched strategies may have the flexibility of using one memory system or the other, some participants may not. In fact, several participants who chose a nonspatial strategy, both healthy participants and patients, reported that they were very poor at finding their way and that they avoid using landmarks in the real world. This means that, at least in some people, the spontaneous use of one strategy over the other is not random. Incidentally, this was also found to be the case in rats: The spatial learners who swam to a previously learned location instead of to the visible platform on a special probe trial of the water maze were also better learners on the allocentric spatial memory task in the water maze (McDonald \& White, 1995).

A genetic predisposition could be translated into an expression that favors higher receptor density, protein synthesis, and blood flow leading to higher amounts of glucose and oxygen in particular brain areas over others (Adams et al., 2001; Nicolle, Bizon, \& Gallagher, 1996). The rats that used a spatial strategy in the plus maze were shown to have a sustained increase in phosphorylated cAMP response element-binding protein (pCREB) in the hippocampus $1 \mathrm{hr}$ after being tested in the plus maze, whereas the rats that used a response strategy instead had an increase in pCREB in the caudate nucleus (Colombo, Brightwell, \& Countryman, 2003). Thus, a particular brain structure could become more efficient at processing information than another through sustained protein synthesis, thus guiding behavior. Alternatively, an experiencedependent bias could have developed in various individuals, acquired over many years or perhaps a lifetime. Because participants found they were good at spatial memory, they continued using it, reinforcing future use of spatial behavior. Support for this idea comes from a study in which the density of gray matter in the posterior part of the hippocampus of taxi drivers was found to be correlated with years of experience (Maguire et al., 2000). In our experiment, participants spontaneously used either spatial memory or nonspatial strategies. They could have done so for several years. With experience, they may have developed a bias, leading them to consistently use a single strategy across several situations. Those individuals who eventually received a medial temporal lobe resection maintained these biases and continued to use a spatial strategy, despite their lesion and poor performance as a result of the lesion. This does not preclude the possibility that an experience-dependent bias rests on the genetic potential to strengthen a given neural pathway. Further research on the contributions of multiple memory systems to navigation is necessary to shed light on the possibilities for intervention in patients who have brain damage (Glisky, 1992).

\section{References}

Abrahams, S., Morris, R. G., Polkey, C. E., Jarosz, J. M., Cox, T. C., Graves, M., \& Pickering, A. (1999). Hippocampal involvement in spatial and working memory: A structural MRI analysis of patients with unilateral mesial temporal lobe sclerosis. Brain and Cognition, 41, 39-65.

Adams, M. M., Smith, T. D., Moga, D., Gallagher, M., Wang, Y., Wolfe, B. B., et al. (2001). Hippocampal dependent learning ability correlates with N-methyl-D-aspartate (NMDA) receptor levels in CA3 neurons of young and aged rats. Journal of Comparative Neurology, 432, 230-243.

Aguirre, G. K., Detre, J. A., Alsop, D. C., \& D'Esposito, M. (1996). The parahippocampus subserves topographical learning in man. Cerebral Cortex, 6, 823-829.

Aguirre, G. K., Zarahn, E., \& D’Esposito, M. (1998). Neural components of topographical representation. Proceedings of the National Academy of Sciences, USA, 95, 839-846.

Badal. (1888). Contribution a l'etude des cecites psychiques. Alexie, agraphie, hemianopsyie inferieure, trouble du sens de l'espace. Archives d'Ophtalmologie, Mars-Avril, 97-117.

Barrash, J. (1998). A historical review of topographical disorientation and its neuroanatomical correlates. Journal of Clinical and Experimental Neuropsychology, 20, 807-827.

Bohbot, V. D., Allen, J. J., \& Nadel, L. (2000). Memory deficits characterized by patterns of lesions to the hippocampus and parahippocampal cortex. Annals of the New York Academy of Sciences, 911, 355-368.

Bohbot, V. D., Kalina, M., Stepankova, K., Spackova, N., Petrides, M., \& Nadel, L. (1998). Spatial memory deficits in patients with lesions to the right hippocampus and to the right parahippocampal cortex. Neuropsychologia, 36, 1217-1238.

Brewer, J. B., Zhao, Z., Desmond, J. E., Glover, G. H., \& Gabrieli, J. D. (1998, August 21). Making memories: Brain activity that predicts how well visual experience will be remembered. Science, 281, 1185-1187.

Collins, D. L., Neelin, P., Peters, T. M., \& Evans, A. C. (1994). Automatic 3D intersubject registration of MR volumetric data in standardized Talairach space. Journal of Computer Assisted Tomography, 18, 192205.

Colombo, P. J., Brightwell, J. J., \& Countryman, R. A. (2003). Cognitive strategy-specific increases in phosphorylated cAMP response elementbinding protein and c-Fos in the hippocampus and dorsal striatum. Journal of Neuroscience, 23, 3547-3554.

Eichenbaum, H., Schoenbaum, G., Young, B., \& Bunsey, M. (1996). Functional organization of the hippocampal memory system. Proceedings of the National Academy of Sciences, USA, 93, 13500-13507.

Eichenbaum, H., Stewart, C., \& Morris, R. G. (1990). Hippocampal representation in place learning. Journal of Neuroscience, 10, 3531-3542.

Ekstrom, A. D., Kahana, M. J., Caplan, J. B., Fields, T. A., Isham, E. A., Newman, E. L., \& Fried, I. (2003, September 11). Cellular networks underlying human spatial navigation. Nature, 425, 184-188.

Epstein, R., \& Kanwisher, N. (1998, April 9). A cortical representation of the local visual environment. Nature, 392, 598-601.

Gaffan, D. (1992). Amnesia for complex naturalistic scenes and for objects following fornix transection in the rhesus monkey. European Journal of Neuroscience, 4, 381-388. 
Glisky, E. L. (1992). Acquisition and transfer of declarative and procedural knowledge by memory-impaired patients: A computer data-entry task. Neuropsychologia, 30, 899-910.

Habib, M., \& Sirigu, A. (1987). Pure topographical disorientation: A definition and anatomical basis. Cortex, 23, 73-85.

Hartley, T., Maguire, E. A., Spiers, H. J., \& Burgess, N. (2003). The well-worn route and the path less traveled: Distinct neural bases of route following and wayfinding in humans. Neuron, 37, 877-888.

Hecaen, H., Tzortzis, C., \& Rondot, P. (1980). Loss of topographic memory with learning deficits. Cortex, 16, 525-542.

Hublet, C., \& Demeurisse, G. (1992). Pure topographical disorientation due to a deep-seated lesion with cortical remote effects. Cortex, 28, 123-128.

Iaria, G., Petrides, M., Dagher, A., Pike, B., \& Bohbot, V. D. (2003). Cognitive strategies dependent on the hippocampus and caudate nucleus in human navigation: Variability and change with practice. Journal of Neuroscience, 23, 5945-5952.

Jarrard, L. E. (1993). On the role of the hippocampus in learning and memory in the rat. Behavioral and Neural Biology, 60, 9-26.

King, J. A., Burgess, N., Hartley, T., Vargha-Khadem, F., \& O'Keefe, J. (2002). Human hippocampus and viewpoint dependence in spatial memory. Hippocampus, 12, 811-820.

Kohler, S., Crane, J., \& Milner, B. (2002). Differential contributions of the parahippocampal place area and the anterior hippocampus to human memory for scenes. Hippocampus, 12, 718-723.

Maguire, E. A., Burgess, N., Donnett, J. G., Frackowiak, R. S., Frith, C. D., \& O'Keefe, J. (1998, May 8). Knowing where and getting there: A human navigation network. Science, 280, 921-924.

Maguire, E. A., Burke, T., Phillips, J., \& Staunton, H. (1996). Topographical disorientation following unilateral temporal lobe lesions in humans. Neuropsychologia, 34, 993-1001.

Maguire, E. A., Frith, C. D., Burgess, N., Donnett, J. G., \& O'Keefe, J. (1998). Knowing where things are: Parahippocampal involvement in encoding object locations in virtual large-scale space. Journal of Cognitive Neuroscience, 10, 61-76.

Maguire, E. A., Gadian, D. G., Johnsrude, I. S., Good, C. D., Ashburner, J., Frackowiak, R. S., \& Frith, C. D. (2000). Navigation-related structural change in the hippocampi of taxi drivers. Proceedings of the National Academy of Sciences, USA, 97, 4398-4403.

Matsumura, N., Nishijo, H., Tamura, R., Eifuku, S., Endo, S., \& Ono, T. (1999). Spatial- and task-dependent neuronal responses during real and virtual translocation in the monkey hippocampal formation. Journal of Neuroscience, 19, 2381-2393.

McDonald, R. J., \& White, N. M. (1995). Hippocampal and nonhippocampal contributions to place learning in rats. Behavioral Neuroscience, 109, 579-593.

Milner, A. D., \& Goodale, M. A. (1995). The visual brain in action. Cambridge, MA: MIT Press.

Milner, B. (1972). Disorders of learning and memory after temporal lobe lesions in man. Clinical Neurosurgery, 19, 421-446.

Mishkin, M., \& Petri, H. L. (1984). Memories and habits: Some implications for the analysis of learning and retention. In L. R. Squire \& N. Butters (Ed.), Neuropsychology of memory (pp. 287-296). New York: Guilford.

Morris, R. G. M., Garrud, P., Rawlins, J. N., \& O’Keefe, J. (1982, June 24). Place navigation impaired in rats with hippocampal lesions. Nature, 297, 681-683.

Nicolle, M. M., Bizon, J. L., \& Gallagher, M. (1996). In vitro autoradiography of ionotropic glutamate receptors in hippocampus and striatum of aged Long-Evans rats: Relationship to spatial learning. Neuroscience, 74, 741-756.
Nunn, J. A., Graydon, F. J., Polkey, C. E., \& Morris, R. G. (1999). Differential spatial memory impairment after right temporal lobectomy demonstrated using temporal titration. Brain, 122, 47-59.

O'Keefe, J., \& Dostrovsky, J. (1971). The hippocampus as a spatial map: Preliminary evidence from unit activity in the freely-moving rat. Brain Research, 34, 171-175.

O'Keefe, J., \& Nadel, L. (1978). The hippocampus as a cognitive map. Oxford, England: Clarendon.

Olton, D. S., \& Papas, B. C. (1979). Spatial memory and hippocampal function. Neuropsychologia, 17, 669-682.

Packard, M. G., \& Knowlton, B. J. (2002). Learning and memory functions of the basal ganglia. Annual Review of Neuroscience, 25, 563-593.

Packard, M. G., \& McGaugh, J. L. (1996). Inactivation of hippocampus or caudate nucleus with lidocaine differentially affects expression of place and response learning. Neurobiology of Learning and Memory, 65, $65-72$.

Pigott, S., \& Milner, B. (1993). Memory for different aspects of complex visual scenes after unilateral temporal- or frontal-lobe resection. Neuropsychologia, 31, 1-15.

Rolls, E. T., Robertson, R. G., \& Georges-Francois, P. (1997). Spatial view cells in the primate hippocampus. European Journal of Neuroscience, 9 , 1789-1794.

Scoville, W. B., \& Milner, B. (1957). Loss of recent memory after bilateral hippocampal lesions. Journal of Neurology, Neurosurgery and Psychiatry, 20, 11-21.

Smith, M. L., \& Milner, B. (1989). Right hippocampal impairment in the recall of spatial location: Encoding deficit or rapid forgetting? Neuropsychologia, 27, 71-81.

Suzuki, W. (1996). Neuroanatomy of the monkey entorhinal, perirhinal and parahippocampal cortices: Organization of the cortical inputs and interconnections with amygdala and striatum. Seminars in the Neurosciences, $8,3-12$.

Talairach, J., \& Tournoux, P. (1988). Co-planar stereotaxic atlas of the human brain. Stuttgart, Germany: Thieme.

Ungerleider, L. G., \& Haxby, J. V. (1994). "What" and "where" in the human brain. Current Opinion in Neurobiology, 4, 157-165.

Van Hoesen, G. W. (1982). The parahippocampal gyrus. Trends in Neuroscience, 5, 345-350.

Vargha-Khadem, F., Gadian, D. G., Watkins, K. E., Connelly, A., Van Paesschen, W., \& Mishkin, M. (1997, July 18). Differential effects of early hippocampal pathology on episodic and semantic memory. Science, 277, 376-380.

Wechsler, D. (1955). Wechsler Adult Intelligence Scale. New York: Psychological Corporation.

White, N. M., \& McDonald, R. J. (2002). Multiple parallel memory systems in the brain of the rat. Neurobiology of Learning and Memory, 77, 125-184.

Worsley, K. J., Liao, C. H., Aston, J., Petre, V., Duncan, G. H., Morales, F., \& Evans, A. C. (2002). A general statistical analysis for fMRI data. NeuroImage, 15, 1-15.

Zola-Morgan, S., Squire, L. R., \& Amaral, D. G. (1986). Human amnesia and the medial temporal region: Enduring memory impairment following a bilateral lesion limited to field CA1 of the hippocampus. Journal of Neuroscience, 6, 2950-2967.

Received August 15, 2003

Revision received November 18, 2003

Accepted December 2, 2003 